\title{
Genome and Epigenome Editing in Mechanistic Studies of Human Aging and Aging-Related Disease
}

\author{
Cia-Hin Lau ${ }^{a}$ e, $f$ Yousin Suh ${ }^{\text {a-d }}$ \\ Departments of a Genetics, ${ }^{\mathrm{b}}$ Ophthalmology and Visual Sciences and ${ }^{\mathrm{C}}$ Medicine and ${ }^{\mathrm{d}}$ Institute for Aging Research, \\ Albert Einstein College of Medicine, Bronx, NY, USA; Departments of ${ }^{\mathrm{e}}$ Mechanical and Biomedical Engineering and \\ fBiology and Chemistry, City University of Hong Kong, Hong Kong, SAR, China
}

\section{Keywords}

Aging · Aging-related disease - CRISPR - Epigenetics . Genetics · Genome editing · Epigenome editing

\begin{abstract}
The recent advent of genome and epigenome editing technologies has provided a new paradigm in which the landscape of the human genome and epigenome can be precisely manipulated in their native context. Genome and epigenome editing technologies can be applied to many aspects of aging research and offer the potential to develop novel therapeutics against age-related diseases. Here, we discuss the latest technological advances in the CRISPRbased genome and epigenome editing toolbox, and provide insight into how these synthetic biology tools could facilitate aging research by establishing in vitro cell and in vivo animal models to dissect genetic and epigenetic mechanisms underlying aging and age-related diseases. We discuss recent developments in the field with the aims to precisely modulate gene expression and dynamic epigenetic landscapes in a spatial and temporal manner in cellular and animal models, by complementing the CRISPR-based editing capability with conditional genetic manipulation tools including chemically inducible expression systems, optogenetics, logic gate genetic circuits, tissue-specific promoters,
\end{abstract}

\section{KARGER}

(C) 2016 S. Karger AG, Basel

E-Mail karger@karger.com

www.karger.com/ger and the serotype-specific adeno-associated virus. We also discuss how the combined use of genome and epigenome editing tools permits investigators to uncover novel molecular pathways involved in the pathophysiology and etiology conferred by risk variants associated with aging and agingrelated disease. A better understanding of the genetic and epigenetic regulatory mechanisms underlying human aging and age-related disease will significantly contribute to the developments of new therapeutic interventions for extending health span and life span, ultimately improving the quality of life in the elderly populations.

(c) 2016 S. Karger AG, Basel

\section{Introduction}

The recent advent of genome and epigenome editing technologies has allowed precise manipulation of the human genome and epigenome by introducing specific changes in native states. Targeted genome editing tools based on zinc finger nuclease (ZFN), transcription activator-like effector nuclease (TALEN), and CRISPR (clus-

\section{Cia-Hin Lau, MS}

Department of Mechanical and Biomedical Engineering

Room Y1618, Academic 1, 83 Tat Chee Avenue

City University of Hong Kong, Kowloon Tong, Hong Kong, SAR (China)

E-Mail lauciahin_4275@yahoo.com

\section{Yousin Suh, $\mathrm{PhD}$}

Department of Genetics, Albert Einstein College of Medicine Michael F. Price Center, 1301 Morris Park Avenue, Room 475 Bronx, NY 10461 (USA)

E-Mail yousin.suh@einstein.yu.edu 
tered regulatory interspaced short palindromic repeat)/ Cas9-based RNA-guided DNA endonuclease have become powerful approaches for modeling human disease by creating isogenic cells and transgenic animals (Fig. 1). These efficient, programmable chimeric nucleases provided a breakthrough in generating genetically engineered cells and animals much faster and more economically than via the traditional homologous recombination-based mutagenic approach [1].

The recent breakthroughs in the CRISPR-based genome editing tool further revolutionize the gene editing technique, due to its simplicity in target design, affordability, high efficiency, versatility, and multiplexing capability [2]. The commonly used CRISPR system can be implemented in mammalian cells by co-expressing Cas 9 nuclease along with single guide RNA (sgRNA), which is derived from a synthetic fusion of the CRISPR RNA array (crRNA) and trans-activating crRNA (tracrRNA) [2] (Fig. 1c). The 20-bp target site sequence of sgRNA immediately needs to be followed by a 3-bp NGG protospacer adjacent motif (PAM) sequence on the $3^{\prime}$ end, and an additional guanine nucleotide on the $5^{\prime}$ end can increase targeting efficiency [2]. The repertoire of CRISPR-mediated tools is expanding fast, and we are now at the dawn of the gene editing age. Substantial improvement of

Fig. 1. Targeted genome and epigenome editing by zinc finger nuclease (ZFN) (a), transcription activator-like effector nuclease (TALEN) (b), and CRISPR (clustered regulatory interspaced short palindromic repeat)/Cas9 systems (c). a ZFN consists of 2 functional domains: a DNA-binding domain that recognizes target genomic DNA sequences by multiple zinc finger modules (ZFs) and a nuclease domain that cleaves target DNA. Each ZF recognizes a unique $3 \mathrm{bp}$ of DNA and is designed to bind specific sequences flanking the desired cleavage site. Two nucleases act as a dimer and introduce a double-strand break (DSB). In the example, 12 nucleotides are targeted by 4 ZFNs, and FokI generates a DSB at the target sites. b A TALEN consists of a nuclear localization signal at the $\mathrm{N}$-terminal, a tandem amino acid repeat domain, and a nuclease (FokI in this figure) fused to the C-terminal. Typically, each repeat contains 33-35 amino acids, including 2 unique residues that recognize a specific DNA sequence. Two TALENs form a complex by binding to genomic DNA in the opposite polarity to ZFNs. c CRISPR/Cas9-based RNA-guided DNA endonuclease (CRISPR/Cas9). A CRISPR/Cas9 complex is a ribonuclear protein comprising CRISPR RNA array (crRNA), trans-activating crRNA (tracrRNA), and an endonuclease (Cas9). crRNA binds with Cas9 and guides the complex to the target DNA. Cas9 creates a DSB through nicking each strand of DNA by HNH and Ruv domains. d CRISPRmediated genome editing for diverse DNA modifications. Upon CRISPR-induced single DSB, the restoration of 2 DNA ends by non-homologous end joining (NHEJ) repair gives rise to small in-
CRISPR's efficiency, specificity, and flexibility following the discovery of CRISPR's variants [3-5], of chemical modifications to the sgRNA [6], and of more efficient delivery methods $[5,7]$ has greatly expanded the CRISPR toolbox. More recently, application of the CRISPR/Cas9 system has expanded beyond genome editing (Fig. 1d) to epigenome engineering (Fig. 1e) to modulate endogenous transcription and epigenetic states [8]. The complement of optogenetics [9] and multi-input logic gate genetic circuits [10] with the CRISPR-based targeted epigenome editing capability permits precise modulation of gene expression and of the epigenetic landscape in a spatial and temporal manner in specific tissues and cells. Epigenome engineering has created another wave of excitement across the scientific research community. Biologists are embracing the power of gene editing tools, particularly the CRISPR/Cas9 system, to explore the landscape of genomes and epigenomes in their native context.

The background and development of ZFN-, TALEN-, and CRISPR-based targeted genome and epigenome editing toolboxes have been extensively reviewed elsewhere $[1,11]$. Here, we discuss the latest major advances in CRISPR-based genome and epigenome editing tools, and the potential application of these programmable chimeric enzymes to aging research. More specifically, we aim to dels in the target DNA and results in gene knockout. The DSB can be repaired by homology-directed repair (HDR) when donor DNA containing the putative functional variant is provided, leading to a desired sequence knock-in. Large genomic DNA deletion and replacement can be generated by the introduction of 2 CRISPR sgRNAs with Cas9 to induce repair of the 2 resultant DSBs by NHEJ and HDR, respectively. A base editing strategy with fusion of CRISPR/dCas9 and a cytidine deaminase enzyme allows programmable editing and irreversible conversion of a target DNA base into another without double-stranded DNA cleavage. Cytidine deamination induces conversion of G:C into an A:T base pair following DNA replication or base excision DNA repair (BER). e CRISPR/dCas9-based precise epigenome engineering for modulating gene transcriptional activities. Gene activation can be achieved by blocking the silencer and insulator regions using dCas9 (catalytically inactive dCas9, D10A/H840A). A stronger gene activation can be achieved by fusing the dCas 9 to a minimal catalytic domain of a chromatin-modifying enzyme such as the VP64 transactivation domain, TET1 DNA demethylase, or p300 histone acetyltransferase. For targeted gene repression, dCas9 can be used to block the insulator and promoter activities. To achieve a gene-silencing effect, dCas9 can be fused with the KRAB transcriptional repressor, DNMT3A DNA methyltransferases, or LSD1 histone demethylase to repress the enhancer and promoter activities of a specific gene. 

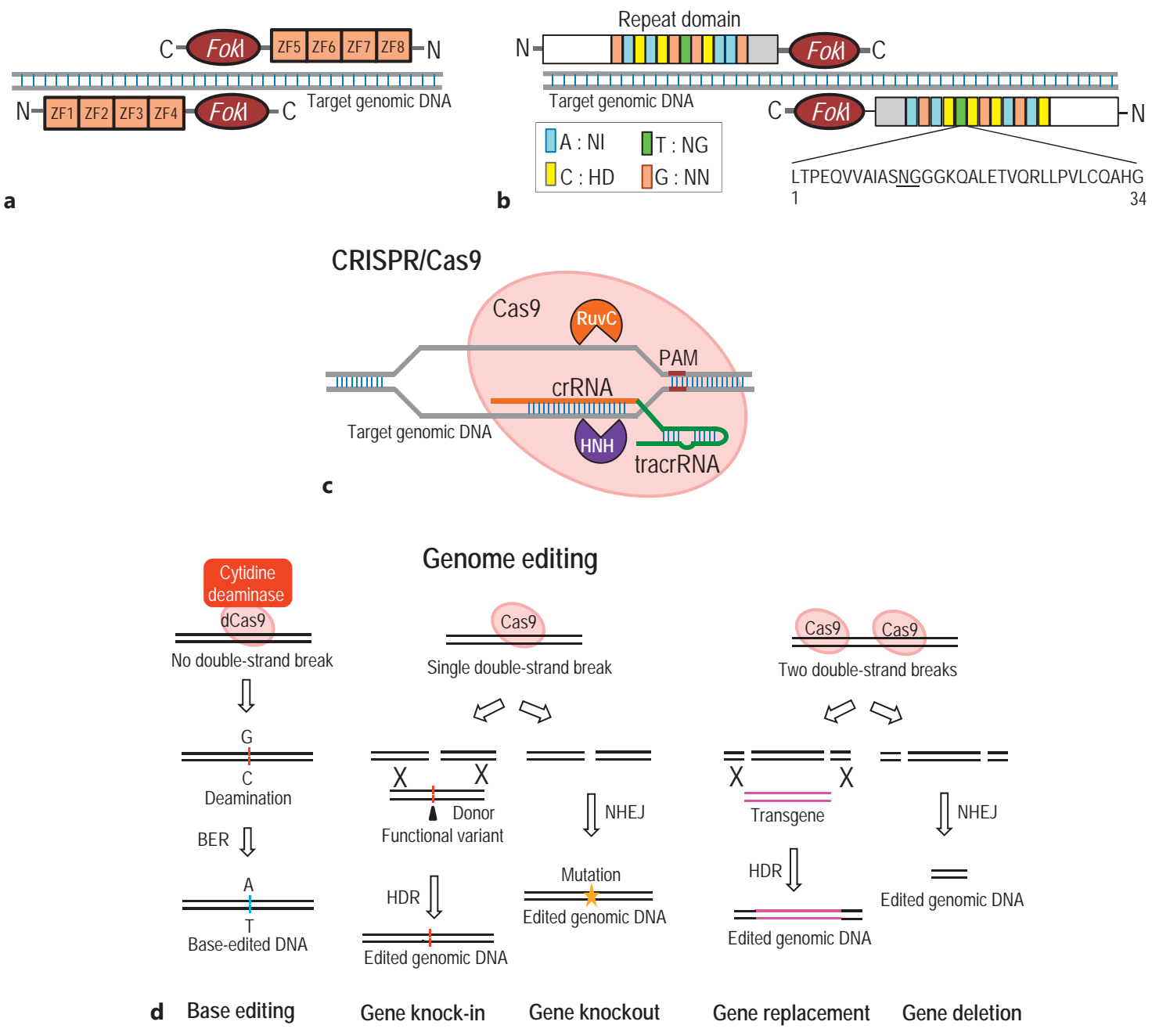

\section{Epigenome editing}

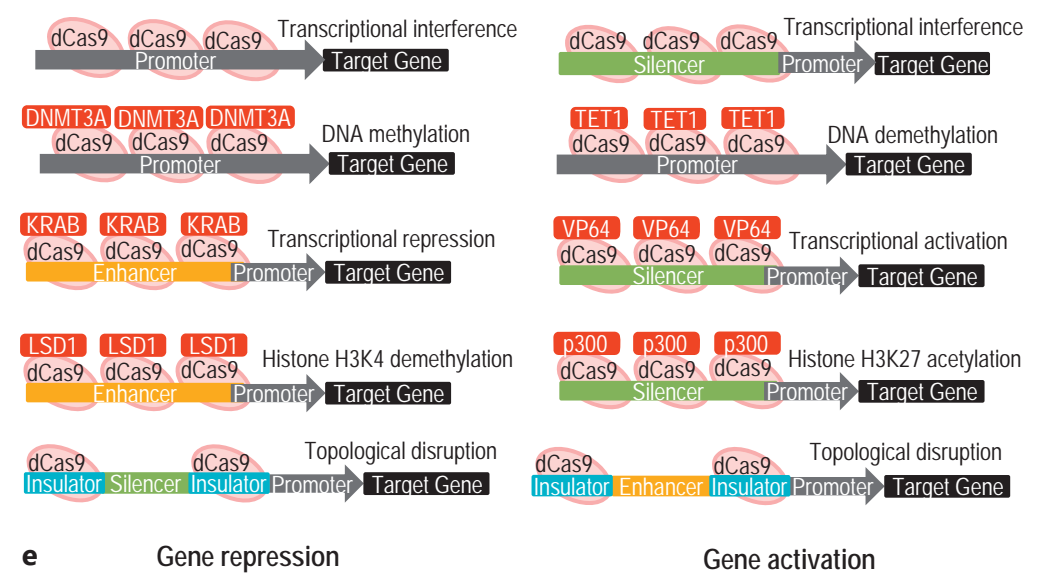


Table 1. Recent advances in CRISPR-based genome editing

\begin{tabular}{l}
\hline Advancement \\
\hline Cas variants (other than wild-type SpCas9) \\
Smaller nuclease size \\
Staphylococcus aureus Cas9 (SaCas9) \\
Streptococcus thermophilus Cas9 (St1Cas9) \\
Controllable sticky end generated \\
Prevotella and Francisella 1 Cas (Cpf1) \\
Class 2 Cas (C2c1) \\
More relaxed PAM \\
Francisella novicida Cas9 (FnCas9) \\
Higher specificity \\
Enhanced-specificity SpCas9 (eSpCas9) \\
High-fidelity SpCas9 (SpCas9-HF1) \\
Double-nicking Cas9 (Cas9 nickase) \\
Targeting single-stranded RNA \\
Leptotrichia shahii Cas (C2c2)
\end{tabular}

Altered PAM specificities to broaden the Cas 9 application Streptococcus pyogenes Cas9 (SpCas9)

Staphylococcus aureus Cas9 (SaCas9)

Altered sgRNA and Cas9 to improve genome editing

efficiency and specificity

Chemically modified sgRNA

Truncated sgRNA

Improved homologous recombination-mediated DNA

replacement and gene knock-in

Asymmetric donor DNA

HDR enhancer

Inhibit NHEJ activity

NHEJ homology-independent knock-in

Silent CRISPR/Cas-blocking mutations

Base editing approach

12

12

3

13

16

17

4

18

14

12

12

Efficient delivery vehicles

Serotype-specific AAV viral vector

Combine use of lipid nanoparticles and AAV viral vectors

5

55

PAM, protospacer adjacent motif; HDR, homology-directed repair; NHEJ, non-homologous end joining; AAV, adenoassociated virus.

provide insight into how these synthetic biology tools could facilitate aging research by establishing in vitro and in vivo models for dissecting genetic and molecular mechanisms underlying age-related diseases.

\section{Recent Major Advances in Genome Engineering}

CRISPR is transforming biomedical science research and has quickly become the preferred tool for genetic manipulation; it also shows incredible promise as a versatile genome editing platform for interrogating endogenous gene function in vitro and in vivo. One of the major additions to the CRISPR toolbox was the discovery of multiple Cas9 variants with expanded capabilities and minimized molecular weight for genetic manipulation to further advance genome and epigenome engineering (Table 1; Fig. 2). Two smaller-sized Cas9 orthologues, Streptococcus thermophilus Cas9 (St1Cas9) and Staphylococcus aureus Cas9 (SaCas9), could edit the genome with an efficiency and specificity similar to those of the commonly used Streptococcus pyogenes Cas9 (SpCas9) $[5,12]$. SaCas9 and its sgRNA expression cassette can be packaged into a single adeno-associated virus (AAV) delivery vehicle for efficient and specific in vivo genome editing [5]. Nevertheless, due to exceeding the maximal viral genome packaging capacity, the addition of tag markers such as the commonly used fluorescent reporter downstream of the fused SaCas 9 and its sgRNA expression cassette results in no production of functional AAV. To circumvent this issue, the previously reported, similar dualvector system can be adopted [7], which uses one vector to express fusion of SaCas9 and fluorescent reporter genes and another to express multiple sgRNAs. In fact, the SpCas9-based dual-vector system was successfully used to interrogate gene function in the mammalian brain by editing multiple genes (Dnmt1, Dnmt3a, and Dnmt3b) in the adult mouse brain in vivo [7]. As illustrated in Figure $2 \mathrm{~b}$, the dual-vector system is particularly useful to deliver large fusion transgenes comprising CRISPR and chromatin catalytic domains as well as including tag markers, multiple guide RNA expression cassettes, optogenetics, or doxycycline-inducible elements. To our knowledge, strategies for in vivo administration of CRISPR-based epigenome editing using AAV vectors have yet to be established.

Recent studies have revealed the potential of another 2 distinct class 2 CRISPR/Cas systems, Cpf1 [3] and C2c1 [13] proteins, which could expand, complement, and extend the existing CRISPR/Cas9 genome editing tools. Cpf1 is a single RNA-guided endonuclease of a class 2 CRISPR/Cas system and could mediate robust and efficient genome editing activity with features distinct from those of Cas9 [3]. Cpf1 lacks tracrRNA, utilizes a T-rich PAM for DNA recognition, and requires only 1 RNA molecule to cleave DNA via a staggered DNA doublestranded break [3]. Instead of "blunt" ends as a result of Cas 9 cutting both strands in a DNA molecule at the same position, the DNA cleavage activity of Cpf1 could create a "sticky" end [3]. The sticky ends carry information that can direct the insertion of the DNA; hence, it makes the 
insertion much better controllable, and this distinct advantage could offer researchers more options when selecting a genome site to edit. This feature allows a better, alternative way to precisely introduce DNA in the proper orientation into the genome via non-homology-directed repair (non-HDR) mechanisms, especially in nondividing cells (Fig. 2a). Apart from Cas9 and Cpf1, C2c1 is another distinct class 2 CRISPR/Cas system, which mediates DNA interference in a $5^{\prime}$-PAM-dependent fashion analogous to Cpf1 [13]. Unlike Cpf1, C2c1 depends on both crRNA and tracrRNA for DNA cleavage [13]. Compared to the RNA-guided DNA-targeting enzymes such as Cpf1 and $\mathrm{C} 2 \mathrm{c} 1, \mathrm{C} 2 \mathrm{c} 2$ is a newly discovered RNA-guided RNAtargeting CRISPR effector, which can be programmed to knock down specific mRNAs by cleaving single-stranded RNA targets carrying complementary protospacers [14].

Engineered CRISPR/Cas9 nucleases with altered PAM specificities eliminate the constraint that only a specific PAM can be recognized by Cas9 [12]. These Cas9 variants with altered PAM specificities allow robust editing of desired endogenous gene sites which are not targetable by wild-type SpCas9 while maintaining the genome-wide specificities comparable to those of wild-type SpCas9 [12]. These Cas9 variants demonstrate the feasibility of engineering a wide range of Cas9s with altered and improved PAM specificities [12]. The SaCas9 PAM sequence for optimal on-target cutting is $5^{\prime}$-NNGRRT-3', which is longer than the $5^{\prime}$-NGG-3' PAM recognition sequence of SpCas9. This short PAM has limited the range of sequences that Cas9 orthologues can target [5]. By using molecular evolution to modify the PAM of SaCas9 in order to relax the PAM recognition specificity, the SaCas9 targeting range can be increased while preserving the robustness of genome editing activities at endogenous loci [15]. Recently, Cas9 from Francisella novicida (FnCas9), one of the largest Cas9 orthologues, was successfully microinjected into mouse zygotes to edit endogenous sites with the more relaxed $5^{\prime}$-YG-3' $3^{\prime}$ PAM, thus expanding the target space of the CRISPR/Cas9 toolbox [16].

Using targeted deep sequencing and unbiased wholegenome off-target analysis, the newly designed "enhanced-specificity" SpCas9 (eSpCas9) variants were shown to reduce off-target effects and maintain robust on-target cleavage in human cells [17]. SpCas9-HF1, another high-fidelity variant of CRISPR/Cas9 nucleases, could significantly reduce unwanted off-target mutations, as shown by genome-wide break capture and targeted sequencing methods [4]. With their exceptional precision and improved specificity, rationally engineered Cas9 nucleases, including SpCas9-HF1 and eSpCas9, could provide an alternative to wild-type SpCas9 for research on and therapeutic applications to numerous inherited disorders $[4,17]$. A higher genome editing specificity while retaining on-target cleavage efficiency could be achieved by combining a Cas9 nickase mutant with paired guide RNAs to introduce targeted double-strand breaks [18]. In addition, use of truncated guide RNAs could further reduce off-target effects induced by pairs of Cas9 nickases without sacrificing on-target genome editing efficiencies [19].

For transgene knock-in or DNA replacement, the efficiency of precise sequence replacement by CRISPR-mediated HDR could be significantly increased using asymmetric donor DNA [20]. The utilization of an HDR enhancer, RS-1 (3-[(benzylamino)sulfonyl]-4-bromo-N[4-bromophenyl]benzamide) was shown to enhance CRISPR/Cas9- and TALEN-mediated knock-in efficiency in rabbit embryos both in vitro and in vivo by stimulating RAD51 [21]. To support this notion, abolished nonhomologous end joining (NHEJ) activity by suppressing the NHEJ key molecules (Ku70 or DNA ligase IV) could increase the efficiency of HDR for CRISPR/Cas9-induced, precise gene editing in mammalian cells [22]. Additionally, highly efficient introduction of specific homozygous and heterozygous mutations could be achieved by incorporating silent CRISPR/Cas-blocking mutations along with pathogenic mutations to improve HDR accuracy [23]. Interestingly, a CRISPR/Cas9-induced NHEJ homology-independent knock-in strategy allows more efficient targeted integration of large reporter genes than does HDR-mediated gene targeting knock-in in human cells [24]. In this strategy, the NHEJ pathway was used to promote DNA integration into a genome by rejoining of cleaved genome and donor plasmids following CRISPR/ Cas9-induced DNA double-strand breaks [24]. The NHEJ homology-independent knock-in strategy is particularly useful for genome editing in postmitotic or nondividing cells, since HDR does not function in $G_{0} / G_{1}$ phase-arrested cells.

More recently, the newly developed base editing approach has permitted programmable editing of a target base in genomic DNA without double-stranded DNA cleavage. This is achieved by directing the irreversible conversion of one target DNA base into another in a programmable manner in the absence of double-stranded DNA backbone cleavage or a donor template [25]. As illustrated in Figure 1d, the base editing strategy involves the use of CRISPR/dCas9 and a cytidine deaminase enzyme fusion for cytidine deamination, followed by DNA replication or base excision DNA repair to mediate con- 


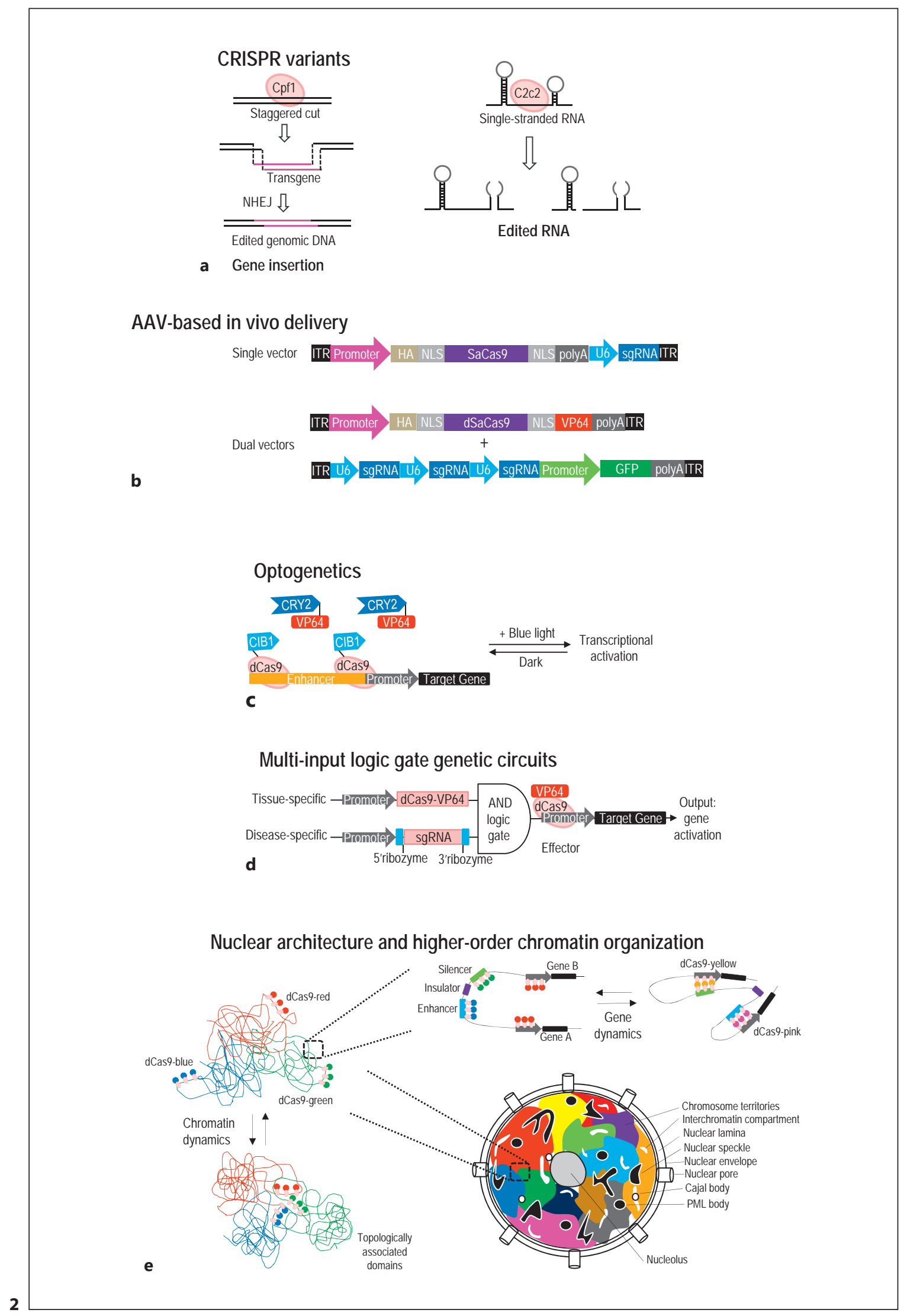

(For legend see next page.) 
version of G:C into an A:T base pair [25]. This scarless genome editing strategy can avoid the issue of random insertions and deletions (indels) at the target locus resulting from the cellular DNA repair response to doublestranded DNA breaks. Hence, this base editing approach has greatly expanded the scope and efficiency of genome editing of point mutations while minimizing indel formation to favor desired clean base editing outcomes. Additionally, co-delivering chemically modified sgRNAs [6] or truncated guide RNAs [19] along with Cas9 mRNA or protein could enhance genome editing efficiency and retain its high specificity by increasing intracellular stability and minimizing the toxicity associated with DNA delivery.

\section{From Genome Engineering to Epigenome Editing}

DNA methylation, histone posttranslational modification, ATP-dependent chromatin remodeling, histone subunit exchange, genomic imprinting, RNA-associated silencing, 3-dimensional (3D) nuclear architecture, and higher-order chromatin organization can contribute to epigenetic alterations and modulate the activities of cisand trans-acting regulatory DNA elements. Epigenetic changes can switch genes on or off and determine which genes are transcribed. The epigenetic marks can be influenced by several factors including age, the environment/ lifestyle, and the disease state. Furthermore, the vast majority of genetic variants associated with the risk of common diseases detected by genome-wide association studies (GWAS) lies in the noncoding regions of the genome [26], suggesting that gene-regulatory changes contribute to interindividual differences in genetic susceptibility to disease. Thus, epigenetic editing tools would be in demand to understand the causal effects of epigenetic variation and ultimately to counteract the epigenetic changes that functionally contribute to disease risk.

Until recently, traditional approaches such as pharmacological inhibition or genetic perturbation have been used to characterize the functional role of cis-/trans-acting DNA regulatory elements and chromatin regulation. These include pharmacological inhibitors of histonemodifying enzymes or epigenetic modifiers such as 50$\mathrm{AzaC}$ and valproic acid and ectopic overexpression or knockdown of these factors followed by genome-wide profiling of gene expression and chromatin states. Obviously, these approaches have limitations in resolving the complex interactions in the native genetic context at the target locus due to the genome-wide changes in chroma-
Fig. 2. Expanding applications with CRISPR variants and combined use of conditional genetic manipulation techniques. a Alternative ways of genetic modification with CRISPR variants. The sticky-end DNA fragments generated by staggered cutting of Cpf1 allow a precise insertion of donor DNA in the proper orientation into the genome via non-homology-directed repair mechanisms such as non-homologous end joining (NHEJ). C2c2 is an RNAguided RNA-targeting CRISPR effector that can be programmed to knock down specific mRNAs by cleaving single-stranded RNA targets carrying complementary protospacers. b In vivo genome and epigenome editing by adeno-associated virus (AAV). SaCas9 and its sgRNA expression cassette can be packaged into a single AAV delivery vehicle for efficient and specific in vivo genome editing. In dual AAV vector systems, dSaCas9-based chromatin modifiers can be used for multiplex epigenome editing by cotransduction of a dSaCas9-VP64 vector and an expression vector with 3 U6-sgRNA cassettes in tandem. c CRISPR/dCas9-based photoactivatable targeted epigenome engineering. In response to blue light irradiation, fusing of sgRNA-dCas9-CIB1 with the light-sensitive CRY2 (cryptochrome 2) bearing VP64 induces targeted gene activation through the VP64 transactivation domain. VP64 colocalizes with dCas9 via CRY2-CIBN interactions and induces transcription only in the presence of blue light. Gene activation is reversible through simple removal of illumination. d CRISPR/dCas9-based AND logic gate genetic circuits. CRISPR/dCas9-based AND gate circuits integrate cellular information from 2 promoters as inputs and activate the output gene only when both inputs are active in the tested disease-relevant cell/tissue types. One promoter (tissuespecific) drives the transcription of dCas9-VP64 mRNA, and another promoter is linked to the transcription of sgRNA targeting a specific gene. The expression of sgRNA is mediated by 2 hammerhead ribozymes placed at both ends of the sgRNA. When the primary transcript is generated, it undergoes self-catalyzed cleavage to release the designed sgRNA. The effector (target gene) can be expressed only when both dCas9-VP64 protein and sgRNA are presented. e CRISPRainbow and multicolor CRISPR for studying nuclear architecture and higher-order chromatin organization. Different fluorescent-tagged dCas9-sgRNAs (red, green, and blue) allow multiplexed labeling of chromatin loci for tracking gene and chromatin dynamics in a spatial and temporal manner, visualizing endogenous genomic loci in live cells. Colocalization of red, green, and blue fluorescents indicates a close physical interaction between topologically associated domains (TADs) from different chromosomes. In the case of studying cis-/trans-acting regulatory elements within a TAD, CRISPRainbow and multicolor CRISPR allow tracking the dynamic interaction of enhancers or silencers with a specific promoter for transcriptional regulation. When the dCas9blue-labeled enhancer is in close contact with the dCas9-redlabeled promoter, gene $\mathrm{A}$ is activated and pink fluorescent can be visualized. Similarly, when the dCas9-green-labeled silencer is in close contact with the dCas9-red-labeled promoter, gene B is activated and yellow fluorescent can be visualized. 
Table 2. Recent advances in CRISPR-based epigenome editing

Advancement

Ref.

Epigenetic marks and chromatin state modulation (dCas 9 nuclease-based fusions)

dCas9 (CRISPRi) (transcriptional interference)

dCas9-VP64 (transcriptional activation)

dCas9-VPR (VP64-p65-Rta) (transcriptional activation)

dCas9-KRAB (Krüppel-associated box) (transcriptional repression)

dCas9-p300 (histone acetylation)

dCas9-LSD1 (histone demethylation)

dCas9-DNMT3A (DNA methylation)

dCas9-peptide repeat and scFv-TET1 catalytic domain fusions (DNA demethylation)

$m R N A$ and protein localization tracking

RCas9 (nuclear-localized RNA-targeting dCas9) (mRNA tracking)

SLENDR (single-cell labeling of endogenous proteins by CRISPR/Cas9-mediated homology-directed repair)

(protein tracking)

Multiplexed labeling of chromatin loci for tracking chromatin dynamics

Multicolor CRISPR (labeling of multiple loci)

CRISPRainbow (labeling of multiple loci)

Reverse information flow from RNA to DNA

RT-Cas1 (reverse transcriptase-Cas1) (reverse transcription)

Scarless genome editing

recCas9 (dCas9-serine recombinase) (genetic recombination)

Orthogonal gene knockout and transcriptional activation

SpCas9 with "dead RNAs" (knock out and activate different genes in the same cell)

60

tin state and gene expression induced by the general, nontargeted manipulation [7]. For many specific histone modifications and for chromatin remodeling, it remains unclear whether these epigenetic states are the cause or the consequence, and these methods do not directly assess causal functional roles for chromatin regulators at specific loci due to the potential pleiotropic effects. Targeted epigenetic editing tools address these issues, allowing investigation of the precise roles played by specific epigenetic modulators on the cell type-specific chromatin state and gene activity in their native contexts.

Cas9 protein has been repurposed by site-specific mutations (D10A; H840A) in the nuclease domain to make nuclease-deficient Cas9, known as "dCas9." CRISPR/ dCas9 has revolutionized the ability to modulate the genomic regulatory elements and epigenetic marks when fused with minimal catalytic domains of chromatinmodifying enzymes to generate synthetic sequence-specific activators and repressors $[8,27]$. The nuclease-based fusions of chromatin-modifying enzymes such as p300 (histone acetyltransferase) [8], LSD1 (histone demethylase or enhancer repressor by removing H3K4 methylation) [27], KRAB (transcriptional repressor) [28], VP64 (transcriptional activator) [29], and TET1 (DNA demethylation) [30] were successfully used to achieve targeted DNA methylation or hydroxymethylation, histone methylation or demethylation, or histone acetylation or deacetylation in mammalian cells (Table 2). A similar strategy could also be adopted for other nucleosomemodulating factors, chromatin remodelers, and histone chaperones to modulate the chromatin structure of genomic functional elements in order to affect the activity of downstream target genes. Hence, targeting histonemodifying activities to specific loci with dCas9 has been a powerful epigenome editing tool for testing the functions of genomic elements and associated chromatin states in their endogenous context [11]. The advances in these nuclease-based site-specific epigenome editing tools have recently been extensively reviewed elsewhere [11].

The establishment and maintenance of the newly introduced epigenetic marks depend on the intrinsic amenability of the target genes, the levels of transcription, the local chromatin context, and the dynamic interplay between chromatin writers, readers, and erasers [11]. A possible approach to maintain heritability of the edited epi- 
genetic marks in the nuclei is by adopting light-inducible CRISPR/dCas9-mediated targeted epigenome engineering. AAV-mediated expression of photoactivatable CRIS$\mathrm{PR} / \mathrm{dCas} 9$ vectors can exist long term as concatemers in nondividing cells; however, this will be lost in replicating cells. To establish conditional knock-in permanent cell lines stably expressing a CRISPR/dCas9-based photoactivatable transcription system, a copy of sgRNA-dCas9CIB1 and transactivation domain-CRY2 (cryptochrome 2) fusion vectors can be inserted into a safe harbor gene knock-in locus such as human AAV integration site 1 and mouse Rosa26 by using a CRISPR/Cas9-mediated homologous recombination approach. In postmitotic cells such as primary neuronal cells in the mammalian brain, there is no need to introduce these optogenetic vectors into the genome, since episomal stability permits longterm transgene expression in nondividing cells. In fact, the nonintegrating and replication-defective nature of AAV is effectively used in optogenetics-based neuroscience experiments to stably express an optogenetic construct in a brain region without the need to integrate these vectors into the host cell genome. As illustrated in Figure $2 \mathrm{c}$, in the presence of blue light, a transactivation domain would colocalize with the catalytically inactive dCas9 following the fusion of the light-sensitive heterodimerizing proteins CRY2 and CIB1 to modulate transcription [9]. Gene activation is reversible through simple removal of illumination. Increasing the number of chromatin-modifying domains localized to the targeted promoter or enhancer sequence could synergistically enhance gene activation [9]. Thus, the CRISPR/dCas9-based photoactivatable transcription system allows rapid and reversible targeted gene activation by light [9]. Since lighting or extinguishing the blue light irradiation is sufficient to switch on or off the epigenomic modulation, this system could be used for precise control of the epigenome editing activity in a spatially and temporally dependent manner for better understanding of the complex gene regulatory networks and chromatin dynamics. Light activation of CRIS$\mathrm{PR} / \mathrm{dCas} 9$ allows for the study of the epigenetic regulatory mechanisms underlying specific gene functions with high precision, and can reduce toxicity from off-target epigenetic modulation by restricting the function of dCas9 to certain locations or time points [9].

To achieve more precise spatial control of transcriptional gene activities, CRISPR/dCas9-based logic gate genetic circuits could be considered. As illustrated in Figure 2d, CRISPR/dCas9-based AND gate circuits can integrate cellular information from 2 promoters as inputs (tissueand disease-specific promoters) and activate the output (target gene expression) only when both inputs are active in the tested cells. Using this chimeric system, the gene of interest can be manipulated to be expressed only in diseased cells of specific tissue origin, but to remain silenced in normal cells or diseased cells of other tissue origin. As a proof of principle, synthesizing AND gate genetic circuits based on CRISPR/Cas9 has been successfully used to specifically identify and control bladder cancer cells in vitro [10]. Theoretically, dCas9 and designed sgRNAs can be used to build any type of transcriptional logic gate and connect them to on-target epigenetic manipulation in living cells and computational analysis of the gene regulatory network. For example, combined use of the elementary logic gates such as AND, OR, NOT, NAND, NOR, $\mathrm{XOR}$, and XNOR (universal symbols for logic gates in electronic fields) allows epigenome manipulation of desired transcriptional regulations at targeted loci. Each of this logic gate uses only 2 inputs (e.g., cell type-dependent and tissue-specific cis-/trans-acting chromatin regulation) to give a distinct output (e.g., epigenetic state and gene expression level). Hence, CRISPR/dCas9-based logic gate genetic circuits could be used to selectively and robustly correct transcriptional misregulation or to restore normal epigenetic patterning in disease-relevant cell types.

Recently, nuclear-localized RNA-targeting nucleaseinactive Cas9 (RCas9) has been developed to track endogenous RNA in living cells in a programmable manner without relying on the incorporation of genetically encoded, exogenous tags [31]. Subsequently, an in vivo genome editing technique called SLENDR (single-cell labeling of endogenous proteins by CRISPR/Cas9-mediated HDR) has been developed to allow high-throughput, high-resolution mapping of protein localization in the mammalian brain [32]. Additionally, an integrated approach using CRISPR genome editing and single-molecule imaging has been used to track the dynamics of telomerase recruitment to telomeres in nuclei of living human cells [33]. These 3 modified CRISPR-based strategies permit real-time tracking of mRNAs and proteins in a specific cellular compartment and live-cell dynamic monitoring of gene and pathway activities.

To understand the dynamic 3D organization and interactions of genomic elements that are critical for the spatial and temporal regulation of gene expression, a series of CRISPR-based imaging techniques has recently been developed (Fig. 2e). These include multicolor CRISPR [34] and CRISPRainbow [35], and they are used to determine genomic DNA localization, dynamics, and interlocus interactions in living cells in space and time. In 
combined use with superresolution microscopy such as structured illumination microscopy or spectral precision distance/position determination microscopy, a CRISPRainbow system allows simultaneous imaging and tracking of multiple genomic loci in living cells to interrogate the intranuclear dynamics and chromatin architecture [35]. As illustrated in Figure 2e, using pairs of differently fluorescent-tagged dCas9-sgRNAs (red, green, and blue), the intranuclear distance between loci on different chromosomes could be determined [34, 35]. CRISPRainbow and multicolor CRISPR also allow tracking the dynamic interaction between cis-/trans-acting regulatory elements and a promoter to rewire transcriptional activity under a specific experimental condition. By correlating the linear intrachromosomal distance and the spatial fluorescence resolution between 2 loci on the same chromosome, the DNA compaction in the region of interest could be assessed in a live cell $[34,35]$. This combination of nanoscale imaging and a CRISPR system opens a new avenue to study the 3D nuclear topography of active and inactive regulatory sequences directly on the individual cell level at a single-molecule resolution.

\section{Genome and Epigenome Engineering to Investigate the Molecular Mechanisms Underlying Aging and Aging-Related Diseases in Humans}

Aging is characterized by a decline in the maintenance of homeostatic processes over time that increases the risk for many common chronic diseases and degenerative conditions, ultimately resulting in death. As aging affects multiple organs and systems in humans, the complexity in animal modeling has been a major obstacle to elucidating the mechanisms by which genetic factors - as seen in hereditary longevity and the high recurrence of the same age-related disease within a family - impact the differential progression of aging. Compared to invertebrate models, vertebrate models (e.g., mice or rats) can recapitulate many more important pathological features of human aging and disease phenotypes. However, modeling aging and age-related diseases in the laboratory is challenging, because classic vertebrate models have relatively long life spans and high costs of maintenance. Luckily, the emerging CRISPR-based genome and epigenome editing technologies have shortened the lengthy steps in generating multiple transgenic animal models. By enabling the introduction of desired genetic modifications in many genes encompassing the hallmarks of aging, the CRISPR/Cas9 technology permits a rapid exploration of their roles in health span and life span in a high-throughput manner as well as a functional characterization of genetic variants mapped by human GWAS [36] (Fig. 3).

The importance of epigenetic dysregulation in the etiology of human disease and the aging process is increasingly recognized. Diverse epigenetic mechanisms have been implicated in human diseases, where it can affect disease etiology and progression. Incorporation of epigenetic variation into genetic studies may help to explain the late onset and progressive nature of most of the common diseases, the severity of their symptoms, the quantitative nature of their complex traits, and the role of the environment in disease development, which a purely sequencebased approach might not be able to do. Even monozygotic twins may succumb to the same disease, albeit often the severity of their symptoms is different and the age at onset is years apart, and this phenotypic discordance has become more noticeable with age; hence, environmental exposure-driven heritable changes in the epigenome play a critical role in the modulation of human aging and aging-related diseases. Cross-sectional and longitudinal data on monozygotic twin pairs have shown that sustained epigenetic differences emerging during the adult life span could contribute to an increasing discordance between monozygotic twins during aging [37]. Apart from genetic defects in genome maintenance and mitochondrial function, epigenetic dysregulation has recently been highlighted as an important biological hallmark of aging and longevity, because transcriptional misregulation as a result of an aberrant epigenetic landscape causes a shorter life span and premature aging in humans and mice [3840]. Genome-wide DNA methylation profiles of centenarians and centenarians' offspring revealed a contribution of epigenetic factors to human aging and longevity [40].

From a clinical point of view, targeted editing of agingrelated genes offers novel therapeutic avenues for multiple diseases. The feasibility of creating specific genetic variants in human induced pluripotent stem cells (iPSCs) derived from patients or engineered de novo has been demonstrated [23]. An iPSC-derived 3D organoid culture system, termed "cerebral organoids" (miniature 3D brain tissue), closely mimics the endogenous developmental program and can serve as a good in vitro model for studying human brain development and a wide range of complex brain diseases [41]. Cerebral organoids can be used in combination with CRISPR/Cas9-based genome and epigenome editing to unravel genetic and epigenetic mechanisms that cause neurodevelopmental and neurodegenerative disorders. Additionally, human primary intestinal stem cells derived from cystic fibrosis patients can 

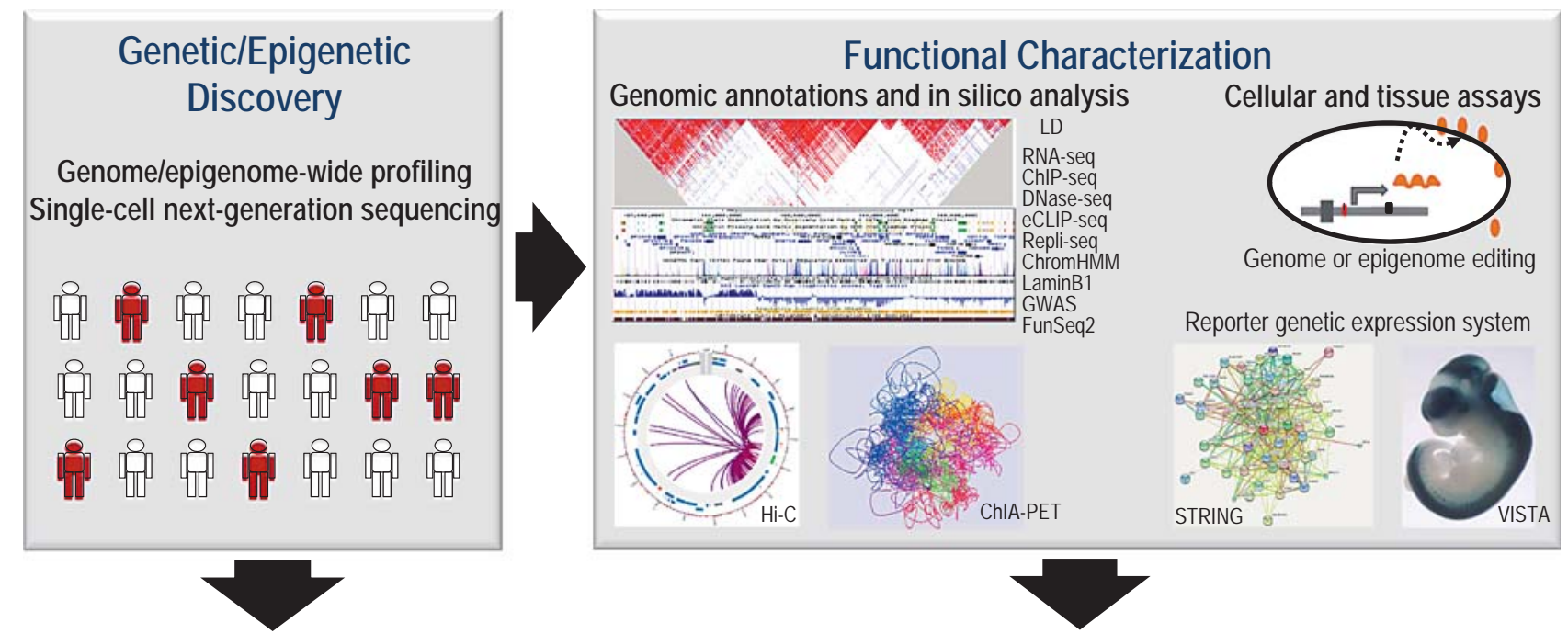

\section{Targeted Medicine of Age-Related Disease}

Diagnostics and prognostics

Genetic disorder screening

Epigenetic landscape monitoring

Intervention and new therapeutics

Cancer immunotherapy

Adult stem cell-based gene therapy

iPSC-based gene therapy

Organoid-based regenerative therapy

Epigenetic-oriented therapy

Personalized medicine

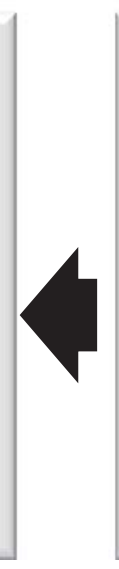

Fig. 3. Translational genetics and epigenetics of aging. A strategy for targeted intervention based on genetic, epigenetic, functional, and mechanistic studies is shown in the flowchart. Genome- and epigenome-wide association studies, chromatin states, and gene expression profiling lead to the identification of genetic variants associated with an aging-related phenotype. Identification of the causal functional variants underlying an association can be further pursued by prioritization through in silico analysis and genome annotations followed by cellular and tissue assays. Bioluminescent- and fluorescent-based reporter genetic expression systems permit high-throughput analysis of functional regulatory variants and intracellular dynamics, followed by prioritization of variants for establishing transgenic cell lines. The VISTA Enhancer Browser allows the identification of distant-acting transcriptional enhancers in the human genome by using a transgenic mouse enhancer assay to characterize noncoding DNA elements with strong evolutionary conservation and epigenomic evidence of putative enhancer marks. Mass spectrometry-based proteomic profiling and functional protein association network (STRING) analysis allow mapping of protein-protein interaction networks. Genome and epigenome editing in induced pluripotent stem cells (iPSCs) and in vivo mouse modeling permit mechanistic studies in the relevant tissue. An iPSC-derived 3D organoid culture system, termed "cerebral organoids" (miniature 3D brain tissue), can serve as a good in vitro model for studying human brain development and a wide range of complex brain diseases. Human primary intestinal stem cells from cystic fibrosis patients can be expanded in culture as genetically and phenotypically stable epithelial organoids for gene correction by CRISPR-mediated homologous recombination. CRISPR-edited immune cells can be used to target and eradicate tumor cells in cancer immunotherapy. The integrated information gained from genetic, epigenetic, functional, and mechanistic studies will ultimately help in the development of effective targeted intervention strategies against aging and aging-related diseases. LD, linkage disequilibrium or haplotype block; RNA-seq, gene expression; ChIP-seq, histone mark enrichment; DNase-seq, open chromatin; eCLIP-seq, RNA-binding protein occupancy; Repli-seq, replication timing; ChromHMM, chromatin state segmentation; LaminB1, nuclear lamina-associated domains; GWAS, genome-wide association studies; FunSeq2, variant annotation; $\mathrm{Hi}-\mathrm{C}$, topologically associating domains and compartments; ChIA-PET, promoter-enhancer links. 
be expanded in culture as genetically and phenotypically stable epithelial organoids for restoring the functional activity of CFTR (cystic fibrosis transmembrane conductance regulator) by CRISPR-mediated homologous recombination [42]. CFTR encodes an anion channel essential for fluid and electrolyte homeostasis of epithelia, and a hereditary defect of this gene causes cystic fibrosis [42]. Since the intestine is an organ with a high turnover and cystic fibrosis is a single-gene hereditary defect disorder, in conjunction with the facts that primary intestinal stem cells can be expanded as cultured organoids and that genetically corrected intestinal organoids can be reimplanted in the intestine of a patient, the precise correction of point mutations linked to cystic fibrosis in patientderived organoids might permit effective adult stem cellbased gene therapy of this disease in the near future. The combined use of a CRISPR/Cas9-based gene correction approach with the organoid culture technology allows clonal expansion of single adult patient stem cells and the selection of genetically modified clonal organoid cultures harboring the precise gene correction (Fig. 3).

Disease modeling in cell lines does not recapitulate the complex interactions between tissues and complex responses to the environment or to drugs. An increasing number of studies have utilized the CRISPR/Cas9 system for in vivo gene editing. Epigenetic studies of longevity and aging based on diverse animal models have recently been comprehensively reviewed [43]. Of special interest is life span extension in various animal models that strongly support an epigenetic role in modulating aging and longevity pathways [43]. Thus far, the majority of evidence suggests histone loss coupled with chromatin remodeling, an imbalance of activating and repressive histone modifications, transcriptional change, global and local change in DNA methylation, site-specific loss of and gain in heterochromatin, and significant nuclear reorganization as a general mechanism of aging in mammalians [43]. For example, to mimic the autism condition in humans, researchers have engineered monkeys to have the MECP2 duplication syndrome [44]. The protein of MECP2 (methyl CpG-binding protein 2) is essential for normal brain function and transcriptional repression through chromatin modification. Severe neurodevelopmental disorders associated with MECP2 include Rett syndrome and X-linked mental retardation [45]. Additionally, many of the symptoms of autism are found in people who have extra copies of the MECP2 gene being expressed in the brain [44]. To examine the impact on autism-like symptoms in monkeys, researchers plan to use the CRISPR system to knock out the extra MECP2 copies in specific brain regions [44]. Depletion of DNA methyltransferase MeCP2 led to lethality and caused a neuronal phenotype, but postnatally, neuron-specific activation of MeCP2 could significantly prolong the life span of mutant mice and delayed the onset of neurologic symptoms [38]. On the other hand, depleting the histone methyltransferase SUV39H1 was shown to improve DNA repair capacity, delay senescence, and extend the life span in a progeria mouse model, providing a potential strategy for aging intervention by targeting SUV39H1-mediated heterochromatin remodeling [39].

The transgenic animal model is useful to recapitulate the premature aging features of the human segmental progeroid syndromes, including Werner syndrome and Hutchinson-Gilford progeria syndrome, which are characterized by defective DNA helicases (WRN) and aberrant processing of the nuclear envelope protein lamin $\mathrm{A}$ (LMNA), respectively [46]. Aberrant DNA methylation profiles in WRN and LMNA mutant patients can also impact these premature aging diseases [46]. By generating human iPSCs from fibroblasts obtained from patients with Hutchinson-Gilford progeria syndrome, in vitro iPSC-based models could recapitulate laminopathy phenotypes such as lack of the nuclear envelope and epigenetic alterations normally associated with premature aging [47]. The CRISPR/Cas9 technology will accelerate the progress in dissecting the contributions of genetic and epigenetic components to life span and longevity in animal models. This gene editing technology allows us to uncover principles that determine the spatial organization of chromosomes, to reveal novel layers of chromatin structure, and to relate these aberrant structures to the gene dysregulation underlying human aging. Modulating chromatin states and cis-/trans-acting DNA regulatory elements may be prime targets for therapeutic intervention to help improve health spans and life spans by correcting transcriptional dysregulation or restore normal epigenetic patterning during aging. Owing to the fact that epigenome editing does not involve genetic changes and the reversible nature of epigenetic mechanisms, it may be less risky with respect to unwanted off-target effects as well as side effects upon healthy cells and tissues.

There have been a number of human clinical trials using alternative gene editing techniques, but none so far has used CRISPR. The application of ZFN technology for gene therapy has been completed, and 6 early-phase clinical trials are currently underway (ClinicalTrials.gov identifiers). Excitingly, the first human trial of CRISPR will start later this year for cancer immunotherapy [48]. A team of researchers from China has announced plans 
to begin the very first clinical trial with CRISPR-edited immune cells as early as August 2016 to treat patients with lung cancer [48]. Other CRISPR trials may not be far behind. In June 2016, an advisory panel of the US National Institutes of Health (NIH) approved a similar CRISPR-based study to create genetically altered immune cells, which are infused back into a patient with melanoma, sarcoma, or myeloma to target and eradicate cancer cells [49]. However, the US proposal has yet to be given the green light from the US Food and Drug Administration (FDA) and a university review board [49]. Although chimeric antigen receptor T-cell therapy was successfully used to treat patients with leukemia [50] and multiple myelomas [51], it did not work for solid tumors such as sarcomas and melanomas. Therefore, CRISPRedited immune cells can be used to overcome the limitation of chimeric antigen receptor-modified $T$ cells to treat patients with solid malignancies.

As the higher-order chromatin structure and heritable epigenetic marks can directly influence gene transcriptional activity, site-specific epigenome editing provides an opportunity to study the contributions of epigenetic regulation to aging and aging-related disease. The CRISPR/Cas9 system can be used to identify and characterize super-enhancers, which are large clusters of transcriptional enhancers with unusually high levels of mediator binding (e.g., cofactors and chromatin regulators) that drive the expression of cell identity genes and, when dysregulated, play a key role in disease [52]. Disease-associated variation was shown to be especially enriched in the super-enhancers of disease-relevant cell types [52]. Insulators and silencers are another 2 important cis-/transacting DNA regulatory elements that may act in concert with enhancers, but with opposing roles in regulating gene transcriptional activity. Chromatin insulators can block the action of transcriptional enhancers by forming chromatin loop domains to affect enhancer-promoter interactions. A recent study revealed that CRISPR-mediated disruption of the CTCF (CCCTC-binding motif) in human IDH wild-type gliomas was able to induce a loss of insulation between topological domains and led to aberrant oncogene activation by permitting a constitutive enhancer to interact aberrantly with a prominent glioma oncogene [53]. Additionally, CRISPR/Cas9 nuclease can be used to disrupt topologically associating domains or chromatin domain clusters - and the boundary regions separating them - to study the consequences of genomic rearrangements in gene dysregulation and disease. Disruption of topologically associating domains was shown to lead to de novo enhancer-promoter interactions and to rewiring of the long-range regulatory genome architecture, thereby affecting the pathogenicity of human structural variants, particularly in noncoding regions of the human genome [54].

\section{Conclusions and Perspective}

Genome editing and epigenome editing have revolutionized biomedical research and offer an unprecedented opportunity to study the molecular mechanisms underlying aging and aging-related diseases in humans. CRISPR expressed by tissue-specific promoters and serotype-specific AAV permits highly precise editing of a genome and an epigenome in a specific tissue. Multi-input logic gate genetic circuits can be integrated with optogenetics-based targeted epigenome engineering to precisely modulate gene expression and the epigenetic landscape in a spatially and temporally specific manner. Light activation of CRISPR could allow gene functional studies with high precision and may reduce toxicity from off-target effects by restricting the function of CRISPR to certain locations (e.g., tissues/cells) and time points (e.g., turn on/off the transcriptional activity). While an increasing number of experimental studies have demonstrated the potential and promises of these techniques, methodological and conceptual advances will be needed before genome and epigenome editing can be effectively and routinely applied as a research tool and in clinical settings. Furthermore, strategies for in vivo delivery of CRISPR/dCas9based epigenome editing tools have yet to be established. The small packaging capacity of AAVs has hindered their application for delivering large fusion transgenes comprising CRISPR/dCas9-based epigenetic modulators.

Together with the genome editing toolbox, site-specific recruitment of engineered chromatin regulators to modulate epigenetic marks and associated chromatin states at proximal promoters or distal DNA regulatory elements will allow us to gain molecular insight into the function of epigenetic marks underlying human aging and aging-related diseases. Combined use of genome and epigenome editing tools will help uncover novel molecular pathways that account for associations detected with global analyses including GWAS by elucidating genotypeepigenotype interactions. A better understanding of the genetic and epigenetic regulatory mechanisms underlying human aging and age-related diseases will significantly contribute to the development of new therapeutic interventions for extending health span and life span, ultimately improving the quality of life of elderly populations. 


\section{Acknowledgments}

This work was funded by NIH grants AG017242, GM104459, and CA180126.

\section{Disclosure Statement}

The authors declare no competing interests.

\section{References}

1 Gaj T, Gersbach CA, Barbas CF 3rd: ZFN, TALEN, and CRISPR/Cas-based methods for genome engineering. Trends Biotechnol 2013;31:397-405.

2 Cong L, Ran FA, Cox D, Lin S, Barretto R, Habib N, Hsu PD, Wu X, Jiang W, Marraffini LA, Zhang F: Multiplex genome engineering using CRISPR/Cas systems. Science 2013;339: 819-823.

3 Zetsche B, Gootenberg JS, Abudayyeh OO, Slaymaker IM, Makarova KS, Essletzbichler P, Volz SE, Joung J, van der Oost J, Regev A, Koonin EV, Zhang F: Cpfl is a single RNAguided endonuclease of a class 2 CRISPR-Cas system. Cell 2015;163:759-771.

4 Kleinstiver BP, Pattanayak V, Prew MS, Tsai SQ, Nguyen NT, Zheng Z, Joung JK: Highfidelity CRISPR-Cas9 nucleases with no detectable genome-wide off-target effects. Nature 2016;529:490-495.

5 Ran FA, Cong L, Yan WX, Scott DA, Gootenberg JS, Kriz AJ, Zetsche B, Shalem O, Wu X, Makarova KS, Koonin EV, Sharp PA, Zhang F: In vivo genome editing using Staphylococcus aureus Cas9. Nature 2015;520:186-191.

6 Hendel A, Bak RO, Clark JT, Kennedy AB, Ryan DE, Roy S, Steinfeld I, Lunstad BD, Kaiser RJ, Wilkens AB, Bacchetta R, Tsalenko A, Dellinger D, Bruhn L, Porteus MH: Chemically modified guide RNAs enhance CRISPRCas genome editing in human primary cells. Nat Biotechnol 2015;33:985-989.

7 Swiech L, Heidenreich M, Banerjee A, Habib N, Li Y, Trombetta J, Sur M, Zhang F: In vivo interrogation of gene function in the mammalian brain using CRISPR-Cas9. Nat Biotechnol 2015;33:102-106.

8 Hilton IB, D'Ippolito AM, Vockley CM, Thakore PI, Crawford GE, Reddy TE, Gersbach CA: Epigenome editing by a CRISPR-Cas9based acetyltransferase activates genes from promoters and enhancers. Nat Biotechnol 2015;33:510-517.

9 Nihongaki Y, Kawano F, Nakajima T, Sato M: Photoactivatable CRISPR-Cas9 for optogenetic genome editing. Nat Biotechnol 2015 33:755-760.

10 Liu Y, Zeng Y, Liu L, Zhuang C, Fu X, Huang $\mathrm{W}$, Cai Z: Synthesizing and gate genetic circuits based on CRISPR-Cas9 for identification of bladder cancer cells. Nat Commun 2014;5:5393.

11 Keung AJ, Joung JK, Khalil AS, Collins JJ: Chromatin regulation at the frontier of synthetic biology. Nat Rev Genet 2015;16:159171.
12 Kleinstiver BP, Prew MS, Tsai SQ, Topkar VV, Nguyen NT, Zheng Z, Gonzales AP, Li Z, Peterson RT, Yeh JR, Aryee MJ, Joung JK: Engineered CRISPR-Cas9 nucleases with altered PAM specificities. Nature 2015;523:481-485.

13 Shmakov S, Abudayyeh OO, Makarova KS, Wolf YI, Gootenberg JS, Semenova E, Minakhin L, Joung J, Konermann S, Severinov K, Zhang F, Koonin EV: Discovery and functional characterization of diverse class 2 CRISPRCas systems. Mol Cell 2015;60:385-397.

14 Abudayyeh OO, Gootenberg JS, Konermann S, Joung J, Slaymaker IM, Cox DB, Shmakov S, Makarova KS, Semenova E, Minakhin L, Severinov K, Regev A, Lander ES, Koonin EV, Zhang F: C2c2 is a single-component programmable RNA-guided RNA-targeting CRISPR effector. Science 2016;353:aaf5573.

15 Kleinstiver BP, Prew MS, Tsai SQ, Nguyen NT, Topkar VV, Zheng Z, Joung JK: Broadening the targeting range of Staphylococcus aureus CRISPR-Cas 9 by modifying PAM recognition. Nat Biotechnol 2015;33:1293-1298.

16 Hirano H, Gootenberg JS, Horii T, Abudayyeh OO, Kimura M, Hsu PD, Nakane T, Ishitani R, Hatada I, Zhang F, Nishimasu H, Nureki O: Structure and engineering of Francisella novicida Cas9. Cell 2016;164:950-961.

17 Slaymaker IM, Gao L, Zetsche B, Scott DA, Yan WX, Zhang F: Rationally engineered Cas9 nucleases with improved specificity. Science 2016;351:84-88.

18 Ran FA, Hsu PD, Lin CY, Gootenberg JS, Konermann S, Trevino AE, Scott DA, Inoue A, Matoba S, Zhang Y, Zhang F: Double nicking by RNA-guided CRISPR Cas9 for enhanced genome editing specificity. Cell 2013; 154:1380-1389.

19 Fu Y, Sander JD, Reyon D, Cascio VM, Joung JK: Improving CRISPR-Cas nuclease specificity using truncated guide RNAs. Nat Biotechnol 2014;32:279-284.

20 Richardson CD, Ray GJ, DeWitt MA, Curie GL, Corn JE: Enhancing homology-directed genome editing by catalytically active and inactive CRISPR-Cas9 using asymmetric donor DNA. Nat Biotechnol 2016;34:339-344.

21 Song J, Yang D, Xu J, Zhu T, Chen YE, Zhang J: RS-1 enhances CRISPR/Cas9- and TALENmediated knock-in efficiency. Nat Commun 2016;7:10548.

22 Maruyama T, Dougan SK, Truttmann MC, Bilate AM, Ingram JR, Ploegh HL: Increasing the efficiency of precise genome editing with CRISPR-Cas9 by inhibition of nonhomologous end joining. Nat Biotechnol 2015;33: 538-542.
23 Paquet D, Kwart D, Chen A, Sproul A, Jacob S, Teo S, Olsen KM, Gregg A, Noggle S, Tessier-Lavigne $\mathrm{M}$ : Efficient introduction of specific homozygous and heterozygous mutations using CRISPR/Cas9. Nature 2016;533 $125-129$.

24 He X, Tan C, Wang F, Wang Y, Zhou R, Cui D, You W, Zhao H, Ren J, Feng B: Knock-in of large reporter genes in human cells via CRISPR/Cas9-induced homology-dependent and independent DNA repair. Nucleic Acids Res 2016;44:e85.

25 Komor AC, Kim YB, Packer MS, Zuris JA, Liu DR: Programmable editing of a target base in genomic DNA without double-stranded DNA cleavage. Nature 2016;533:420-424.

26 Roadmap Epigenomics Consortium, Kundaje A, Meuleman W, Ernst J, Bilenky M, Yen A, Heravi-Moussavi A, et al: Integrative analysis of 111 reference human epigenomes. Nature 2015;518:317-330.

27 Kearns NA, Pham H, Tabak B, Genga RM, Silverstein NJ, Garber M, Maehr R: Functional annotation of native enhancers with a Cas9histone demethylase fusion. Nat Methods 2015;12:401-403

28 Mandegar MA, Huebsch N, Frolov EB, Shin E, Truong A, Olvera MP, Chan AH, Miyaoka Y, Holmes K, Spencer CI, Judge LM, Gordon DE, Eskildsen TV, Villalta JE, Horlbeck MA, Gilbert LA, Krogan NJ, Sheikh SP, Weissman JS, Qi LS, So PL, Conklin BR: CRISPR interference efficiently induces specific and reversible gene silencing in human iPSCs. Cell Stem Cell 2016;18:541-553.

29 Hu J, Lei Y, Wong WK, Liu S, Lee KC, He X, You W, Zhou R, Guo JT, Chen X, Peng X, Sun $\mathrm{H}$, Huang $\mathrm{H}$, Zhao H, Feng B: Direct activation of human and mouse Oct4 genes using engineered TALE and Cas9 transcription factors. Nucleic Acids Res 2014;42:4375-4390.

30 Morita S, Noguchi H, Horii T, Nakabayashi K, Kimura M, Okamura K, Sakai A, Nakashima H, Hata K, Nakashima K, Hatada I: Targeted DNA demethylation in vivo using dCas9-peptide repeat and scFv-TET1 catalytic domain fusions. Nat Biotechnol 2016;34: 1060-1065.

31 Nelles DA, Fang MY, O’Connell MR, Xu JL, Markmiller SJ, Doudna JA, Yeo GW: Programmable RNA tracking in live cells with CRISPR/Cas9. Cell 2016;165:488-496.

32 Mikuni T, Nishiyama J, Sun Y, Kamasawa N, Yasuda R: High-throughput, high-resolution mapping of protein localization in mammalian brain by in vivo genome editing. Cell 2016;165:1803-1817. 
33 Schmidt JC, Zaug AJ, Cech TR: Live cell imaging reveals the dynamics of telomerase recruitment to telomeres. Cell 2016;166:11881197.e9.

$34 \mathrm{Ma} \mathrm{H}$, Naseri A, Reyes-Gutierrez P, Wolfe SA, Zhang S, Pederson T: Multicolor CRISPR labeling of chromosomal loci in human cells. Proc Natl Acad Sci USA 2015;112:3002-3007.

$35 \mathrm{Ma} \mathrm{H}$, Tu LC, Naseri A, Huisman M, Zhang S, Grunwald D, Pederson T: Multiplexed labeling of genomic loci with dCas9 and engineered sgRNAs using CRISPRainbow. Nat Biotechnol 2016;34:528-530.

36 Harel I, Benayoun BA, Machado B, Singh PP, Hu CK, Pech MF, Valenzano DR, Zhang E, Sharp SC, Artandi SE, Brunet A: A platform for rapid exploration of aging and diseases in a naturally short-lived vertebrate. Cell 2015; 160:1013-1026.

37 Talens RP, Christensen K, Putter H, Willemsen G, Christiansen L, Kremer D, Suchiman HE, Slagboom PE, Boomsma DI, Heijmans BT: Epigenetic variation during the adult lifespan: cross-sectional and longitudinal data on monozygotic twin pairs. Aging Cell 2012; 11:694-703.

38 Giacometti E, Luikenhuis S, Beard C, Jaenisch R: Partial rescue of MeCP2 deficiency by postnatal activation of MeCP2. Proc Natl Acad Sci USA 2007;104:1931-1936.

39 Liu B, Wang Z, Zhang L, Ghosh S, Zheng H, Zhou Z: Depleting the methyltransferase Suv39h1 improves DNA repair and extends lifespan in a progeria mouse model. Nat Commun 2013;4:1868.

40 Gentilini D, Mari D, Castaldi D, Remondin D, Ogliari G, Ostan R, Bucci L, Sirchia SM Tabano S, Cavagnini F, Monti D, Franceschi C, Di Blasio AM, Vitale G: Role of epigenetics in human aging and longevity: genome-wide DNA methylation profile in centenarians and centenarians' offspring. Age (Dordr) 2013;35: 1961-1973.

41 Lancaster MA, Renner M, Martin CA, Wenzel D, Bicknell LS, Hurles ME, Homfray T, Penninger JM, Jackson AP, Knoblich JA: Cerebral organoids model human brain development and microcephaly. Nature 2013;501: 373-379.

42 Schwank G, Koo BK, Sasselli V, Dekkers JF, Heo I, Demircan T, Sasaki N, Boymans S,
Cuppen E, van der Ent CK, Nieuwenhuis EE, Beekman JM, Clevers H: Functional repair of CFTR by CRISPR/Cas9 in intestinal stem cell organoids of cystic fibrosis patients. Cell Stem Cell 2013;13:653-658.

43 Sen P, Shah PP, Nativio R, Berger SL: Epigenetic mechanisms of longevity and aging. Cell 2016;166:822-839.

44 Cyranoski D: Monkeys genetically modified to show autism symptoms. Nature 2016;529: 449 .

45 Meloni I, Bruttini M, Longo I, Mari F, Rizzolio F, D'Adamo P, Denvriendt K, Fryns JP, Toniolo D, Renieri A: A mutation in the Rett syndrome gene, MECP2, causes X-linked mental retardation and progressive spasticity in males. Am J Hum Genet 2000;67:982-985.

46 Heyn H, Moran S, Esteller M: Aberrant DNA methylation profiles in the premature aging disorders Hutchinson-Gilford progeria and Werner syndrome. Epigenetics 2013;8:28-33.

47 Liu GH, Barkho BZ, Ruiz S, Diep D, Qu J, Yang SL, Panopoulos AD, Suzuki K, Kurian L, Walsh C, Thompson J, Boue S, Fung HL, Sancho-Martinez I, Zhang K, Yates J 3rd, Izpisua Belmonte JC: Recapitulation of premature ageing with iPSCs from HutchinsonGilford progeria syndrome. Nature 2011;472: 221-225.

48 Cyranoski D: Chinese scientists to pioneer first human CRISPR trial. Nature 2016;535: 476-477.

49 First-in-human CRISPR trial. Nat Biotechnol 2016;34:796

50 Grupp SA, Kalos M, Barrett D, Aplenc R, Porter DL, Rheingold SR, Teachey DT, Chew A, Hauck B, Wright JF, Milone MC, Levine BL, June $\mathrm{CH}$ : Chimeric antigen receptor-modified $\mathrm{T}$ cells for acute lymphoid leukemia. $\mathrm{N}$ Engl J Med 2013;368:1509-1518.

51 Garfall AL, Maus MV, Hwang WT, Lacey SF, Mahnke YD, Melenhorst JJ, Zheng Z, Vogl DT, Cohen AD, Weiss BM, Dengel K, Kerr ND, Bagg A, Levine BL, June CH, Stadtmauer EA: Chimeric antigen receptor $T$ cells against CD19 for multiple myeloma. N Engl J Med 2015;373:1040-1047.

52 Hnisz D, Abraham BJ, Lee TI, Lau A, SaintAndré V, Sigova AA, Hoke HA, Young RA: Super-enhancers in the control of cell identity and disease. Cell 2013;155:934-947.
53 Flavahan WA, Drier Y, Liau BB, Gillespie SM, Venteicher AS, Stemmer-Rachamimov AO, Suvà ML, Bernstein BE: Insulator dysfunction and oncogene activation in IDH mutant gliomas. Nature 2016;529:110-114.

54 Lupiáñez DG, Kraft K, Heinrich V, Krawitz P, Brancati F, Klopocki E, Horn D, Kayserili H Opitz JM, Laxova R, Santos-Simarro F, Gilbert-Dussardier B, Wittler L, Borschiwer M, Haas SA, Osterwalder M, Franke M, Timmermann B, Hecht J, Spielmann M, Visel A, Mundlos S: Disruptions of topological chromatin domains cause pathogenic rewiring of gene-enhancer interactions. Cell 2015;161: 1012-1025.

55 Yin H, Song CQ, Dorkin JR, Zhu LJ, Li Y, Wu Q, Park A, Yang J, Suresh S, Bizhanova A, Gupta A, Bolukbasi MF, Walsh S, Bogorad RL, Gao G, Weng Z, Dong Y, Koteliansky V, Wolfe SA, Langer R, Xue W, Anderson DG: Therapeutic genome editing by combined viral and non-viral delivery of CRISPR system components in vivo. Nat Biotechnol 2016;34: 328-333.

56 Chavez A, Scheiman J, Vora S, Pruitt BW, Tuttle M, Iyer E, Lin S, Kiani S, Guzman CD Wiegand DJ, Ter-Ovanesyan D, Braff JL, Davidsohn N, Housden BE, Perrimon N, Weiss R, Aach J, Collins JJ, Church GM: Highly efficient Cas9-mediated transcriptional programming. Nat Methods 2015;12:326-328.

57 Vojta A, Dobrinić P, Tadić V, Bočkor L, Korać P, Julg B, Klasić M, Zoldoš V: Repurposing the CRISPR-Cas9 system for targeted DNA methylation. Nucleic Acids Res 2016;44: 5615-5628.

58 Silas S, Mohr G, Sidote DJ, Markham LM, Sanchez-Amat A, Bhaya D, Lambowitz AM, Fire AZ: Direct CRISPR spacer acquisition from RNA by a natural reverse transcriptase-Cas 1 fusion protein. Science 2016;351:aad4234.

59 Chaikind B, Bessen JL, Thompson DB, Hu JH, Liu DR: A programmable Cas9-serine recombinase fusion protein that operates on DNA sequences in mammalian cells. Nucleic Acids Res 2016, Epub ahead of print.

60 Dahlman JE, Abudayyeh OO, Joung J, Gootenberg JS, Zhang F, Konermann S: Orthogonal gene knockout and activation with a catalytically active Cas9 nuclease. Nat Biotechnol 2015;33:1159-1161. 